\title{
Potencial de SIG participativos na Gestão de Riscos de Desastres e Emergências em Saúde
}

\author{
Potential of participatory GIS in Disaster Risk Management and \\ Health Emergencies
}

Leonardo Esteves de Freitas $\mathbf{1 , 2 , 3}$, Flávio Souza Brasil Nunes, $\mathbf{1 , 2}, \mathbf{3}$

DOI: 10.1590/0103-11042020E215

RESUMO A Gestão de Riscos de Desastres e Emergências em Saúde (GRDES) constitui um dos temas importantes na Agenda 2030. No Brasil, apresenta contornos agudos, tendo em vista que a ocorrência e magnitude desses eventos têm crescido. Grandes esforços e investimentos voltados para a Gestão de Riscos de Desastres vêm sendo realizados. Um elemento fundamental é incorporar o conhecimento popular nos modelos de análise. Nesse sentido, moradores de Nova Friburgo, instituições de ensino e/ou pesquisa e poder público criaram, em 2014, uma rede colaborativa com objetivo de reduzir riscos de desastres. Uma das ações preconizadas por essa rede é a construção de um Sistema de Informação Geográfica (SIG) capaz de apoiar as atividades de GRDES. O presente trabalho discute a construção dessa base de dados. Foram realizados esforços em levantamentos, organização e sistematização de informações geográficas de diversas fontes e escalas, além de terem sido aplicadas técnicas de mapeamento participativo para levantamento de informações com moradores. Como resultado, esse SIG tornou-se uma ferramenta de integração entre saberes científicos e populares capaz de subsidiar o conjunto de processos relacionados com a GRDES em escala local.

PALAVRAS-CHAVE Sistema de informação geográfica. Gestão de riscos. Pesquisa participativa baseada na comunidade.

1 Universidade Federal do Rio de Janeiro (UFRJ), Laboratório de Geohidroecologia e Gestão de Riscos (Geoheco) - Rio de Janeiro (RJ), Brasil. leonardofreitas73@gmail. com

2 Fundação Oswaldo Cruz (Fiocruz), Escola Nacional de Saúde Pública Sergio Arouca (Ensp), Centro de Estudos e Pesquisas em Desastres e Emergências em Saúde (Cepedes) - Rio de Janeiro (RJ), Brasil.

${ }^{3}$ Fundação Oswaldo Cruz (Fiocruz), Observatório de Territórios Sustentáveis e Saudáveis da Bocaina (OTSS) - Rio de Janeiro (RJ), Brasil.

\begin{abstract}
Disaster Risk Management and Health Emergencies is one of the important themes in the 2030 Agenda. In Brazil, it presents acute contours, considering that the occurrence and magnitude of these events have grown. Major efforts and investments focused on Disaster Risk Management have been made. A fundamental element is to incorporate popular knowledge in the models of analysis. In this sense, residents of Nova Friburgo, teaching institutions and/or research and public authorities created, in 2014, a collaborative network with the objective of reducing disaster risks. One of the actions recommended by this network is the construction of a Geographic Information System (GIS) capable of supporting the activities of risk management of disasters and health emergencies. The present work discusses the construction of this database. Efforts were made to survey, organize and systematize geographic information from different sources and scales, in addition to participatory mapping techniques for gathering information from residents. As a result, this GIS has become a tool for integrating scientific and popular knowledge capable of subsidizing the set of processes related to Disaster Risk Management and Health Emergencies at a local scale.
\end{abstract}

KEYWORDS Geographic information systems. Risk management. Community-based participatory research. 


\section{Introdução}

A 'Agenda 2030' (A2030) da Organização das Nações Unidas incluiu a Gestão de Riscos de Desastres (GRD) como um de seus elementos fundamentais, especialmente a partir do Objetivo de Desenvolvimento Sustentável(ODS) número 11: Cidades e Comunidades Sustentáveis - que busca reduzir mortes e pessoas afetadas por desastres, focalizando proteger as pessoas que se encontram em situação vulnerável'. Preconiza a adoção e implementação de políticas e planos integrados para a mitigação e adaptação às mudanças climáticas e à resiliência a desastres, desenvolvendo um processo de gerenciamento holístico do risco de desastres em acordo com o Marco de Sendai para a Redução do Risco de Desastres 2015-2030.

O ODS 3 também possui aderência à GRD, especialmente a partir da meta $3 \mathrm{~d}$, voltada para o desenvolvimento de alertas precoces e redução e gerenciamento de riscos nacionais e globais de saúde, principalmente em países em desenvolvimento.

A implantação de um processo integrado de GRD e emergências em saúde, preconizados pelos dois ODS acima referidos, depende da construção de modelos de GRD que garantam a integração dos processos de gestão.

De modo geral, os modelos de GRD partem de duas concepções. Existem aqueles tradicionais, que pressupõem uma concepção linear, na qual a temporalidade implica a impossibilidade de retorno às condições iniciais, em um processo unidirecional, que parte do passado para o futuro ${ }^{2,3}$. Há modelos que pressupõem uma concepção circular do tempo, em que as fases da GRD se apresentam como processos integrados e interdependentes: as etapas que ocorrem no período anterior ao desastre influenciam aquelas que ocorrem pós-desastre; $\mathrm{e}$ estas últimas influenciam as etapas anteriores, na medida em que geram novas vulnerabilidades e exacerbam ou reduzem vulnerabilidades existentes $^{\mathbf{3}, \mathbf{4}}$. Inclusive, os modelos circulares de GRD têm sido discutidos pela Defesa Civil brasileira $^{5}$, especialmente após o desastre da
Região Serrana de 2011, quando uma mudança na legislação sobre o tema teve impulso no País ${ }^{6}$. A concepção circular representa um avanço em direção ao que preconiza a A2030 e o 'Marco de Sendai', pois possibilita compreender que a gestão deve ser integrada e contínua e que todas as etapas influenciam as demais, direcionando a gestão para uma perspectiva de processos.

Sob essa perspectiva, a GRD deve ser estruturada a partir de seis processos fundamentais:

1) gerar conhecimento sobre risco de desastre em seus diferentes âmbitos, 2) prevenir riscos futuros, 3) reduzir riscos existentes, 4) preparar a resposta ao desastre, 5) responder e reabilitar e 6) recuperar e reconstruir $\mathbf{7 ( 6 2 )}$.

Os processos de resposta e reabilitação e de recuperação e reconstrução devem ser explorados sob a perspectiva de gerar conhecimentos para a gestão e prevenir riscos futuros e atuais, garantindo a continuidade e a integração nos processos de GRD. Narváez et al. resumem esse enfoque:

[...] os processos de intervenção não devem ser entendidos como independentes ou separados, na verdade eles são contínuos [...], são interdependentes (por exemplo, prevenção ou redução de risco não podem ser entendidas sem existir previamente gestão do conhecimento de riscos de desastre) ${ }^{\mathbf{7}(61)}$.

\section{Geração de conhecimento e levantamento de informações}

Na perspectiva de uma gestão de riscos contínua e integrada, a geração de conhecimento é um processo que dá mais consistência às demais etapas da GRD ${ }^{7}$.

O 'Marco de Sendai'8 define como prioridade compreender o risco de desastres em todas 
as suas dimensões e utilizar esse conhecimento para avaliar o risco previamente, mitigar os impactos e aplicar medidas adequadas de preparação e resposta. E afirma que:

Abordar os fatores subjacentes de risco de desastres através de investimentos públicos e privados informados sobre riscos é mais custo-eficiente do que concentrar-se principalmente em resposta pós-desastres e recuperação, contribuindo, também, para o desenvolvimento sustentáve|8(9).

No entanto, algumas questões fundamentais devem ser respondidas para que o processo de geração de conhecimento seja capaz de subsidiar a gestão de riscos e emergências em saúde relacionadas com os desastres:

- Qual conhecimento deve ser gerado e/ou levantado e disponibilizado?

- Diferentes escalas de gestão de riscos e diferentes contextos requerem geração de conhecimentos ou levantamento de informações distintas?

- Como armazenar e disponibilizar esse conhecimento, considerando as etapas do processo de gestão de riscos e recortes/escalas espaciais de gestão?

Diversos autores têm trabalhado essas questões, também abordadas pelo 'Marco de Sendai'. Em relação a que conhecimentos gerar e informações levantar, alguns são considerados essenciais em diferentes contextos. $\mathrm{O}$ levantamento das ameaças existentes em um determinado território; o conhecimento das vulnerabilidades dos diferentes segmentos da população e das instituições envolvidas com GRD; o nível de exposição da população, especialmente dos segmentos mais vulneráveis; e, por fim, o conhecimento da capacidade de resposta dos diferentes setores envolvidos na gestão de riscos são essenciais para a GRD711. No caso específico da gestão de emergências em saúde, o conhecimento detalhado da capacidade de resposta do setor saúde em seus diversos componentes é indispensável ${ }^{9}$.

\section{Sistemas de Informação Geográfica no apoio à gestão de riscos}

A utilização de geotecnologias apoiadas em Sistemas de Informação Geográfica (SIG) com o foco na Gestão de Riscos de Desastres e Emergências em Saúde (GRDES) potencializa os processos de gestão. Essa indicação, inclusive, é colocada como um dos princípios norteadores do 'Marco de Sendai', que inclui os SIG como estratégia para melhorar as ferramentas de medição e a coleta, a análise e a divulgação dos dados ${ }^{8}$.

Os SIG permitem a integração, em uma única base de dados, de informações geográficas provenientes de fontes e formatos diversos, oferecendo mecanismos para recuperar, manipular e visualizar essas informações ${ }^{12}$. Portanto, facilitam o levantamento, o armazenamento, a análise e a disponibilização de grande quantidade de dados que, devido à complexidade dos desastres e das emergências em saúde, seriam inviáveis de serem tratados utilizando métodos analógicos e/ou tradicionais ${ }^{13}$.

Os SIG, portanto, oferecem ganhos consideráveis nos processos associados à GRDES, incluindo planejamento para redução de riscos, definição de medidas de preparação e resposta, implementação de ações de recuperação e reabilitação e tomada de decisões emergenciais ${ }^{13}$. O mapeamento de vulnerabilidades e capacidades de resposta, por exemplo, considerado por Freitas et al. ${ }^{9}$ como uma das etapas fundamentais para a GRDES, pode ser realizado com apoio dos SIG, ganhando em agilidade, integração de dados e ampliando a possibilidade de comunicação. A utilização de um SIG pode se apoiar na identificação das Unidades de Saúde (US) aptas a receber a população em casos de emergência, na análise da evolução espaço-temporal de doenças e agravos, na localização 
de pessoas vulneráveis e no reconhecimento de áreas 'seguras' para a realocação de comunidades afetadas ${ }^{13}$, entre outras.

Os SIG permitem combinar informações disponíveis em órgãos públicos e/ou levantadas em pesquisas específicas. Assim, como relacionar o número de leitos de urgência e emergência e o número de pessoas vulneráveis em um determinado recorte territorial. Essas combinações oferecem inúmeras possibilidades de avaliações e análises espaciais, indispensáveis para o planejamento territorial e para a GRDES.

Outro aspecto importante da utilização dos SIG é à facilidade desses sistemas em elaborar mapas temáticos, que possuem grande potencial para apoiar a comunicação de risco.

Construir os SIG para apoiar a GRDES em diversas escalas e recortes espaciais, por conseguinte, é fundamental e fortalece significativamente a capacidade de gestão.

\section{Os SIG e a gestão participativa de riscos}

O conhecimento que as comunidades que vivem em áreas de risco possuem sobre seus territórios é essencial para a GRD. Em seus princípios orientadores, o Marco de Sendai afirma que a Redução de Riscos de Desastres (RRD) requer que as informações sobre riscos estejam acessíveis, atualizadas, inteligíveis e que tenham base científica, mas que sejam complementadas com os conhecimentos tradicionais, ou seja, populares ${ }^{8}$.

Isso é particularmente importante quando o processo de gestão está voltado para a escala local. Conhecer as vulnerabilidades, ameaças, níveis de exposição e capacidades de resposta das comunidades é fundamental, e a melhor forma de fazer isso é no contato com as comunidades ${ }^{\mathbf{1 4}-16}$.

A utilização dos SIG é uma alternativa, a despeito de ser uma ferramenta que, até pouco tempo, não era utilizada com essa finalidade. Durante muitos anos, os SIG tinham sua utilização relacionada com processos de vigilância social, reforçando as relações entre conhecimento e poder e o controle de populações. Nos últimos 20 anos, surgiram trabalhos de construção dos SIG a partir de um modelo participativo, que inclui as comunidades na construção desses sistemas, em contraposição ao modelo tradicional17.

A partir dessa concepção, os SIG se tornaram ferramentas para a GRD na escala local, especialmente para o levantamento de informações nas comunidades e para o armazenamento das informações comunitárias e disponibilização das informações técnico-científicos para as comunidades ${ }^{17,18}$.

O envolvimento das comunidades locais nos levantamentos das informações fortalece esses sistemas, pois diversas caraterísticas essenciais dos territórios só os moradores conhecem ${ }^{\mathbf{1 8 - 2 0}}$.

Ademais, construir um SIG com as comunidades, a partir de uma perspectiva participativa, é uma estratégia que contribui para um diálogo entre os saberes técnico-científicos, institucionais e comunitários, facilitando o desenvolvimento de agendas voltadas aos interesses e ao território dessas comunidades ${ }^{21-23}$.

\section{Reger Córrego d'Antas}

Essa forma de construir os SIG está sendo colocada em prática no âmbito da Rede para a Gestão de Riscos da Bacia do Córrego d'Antas (Reger-CD), que vem sendo estruturada por instituições de pesquisa e gestão e por comunidades que vivem na bacia hidrográfica do Córrego d'Antas ${ }^{20}$. Essa bacia, situada no município de Nova Friburgo, Região Serrana do estado do Rio de Janeiro (figura 1), foi intensamente atingida no evento catastrófico de janeiro de 2011. Dezenas de mortes ocorreram nessa área, além de prejuízos materiais incomensuráveis.

Esse desastre marcou toda a Região Serrana Fluminense. Induzidos por chuvas extremas, ocorreram milhares de deslizamentos em Petrópolis, Teresópolis e Nova Friburgo, bem como enxurradas e enchentes nos municípios situados a jusante ${ }^{24}$. Oficialmente, esse desastre levou a óbito 964 pessoas, sendo $448 \mathrm{em}$ Nova Friburgo 25 
Figura 1. Localização da Bacia Hidrográfica de Córrego d'Antas, no município de Nova Friburgo
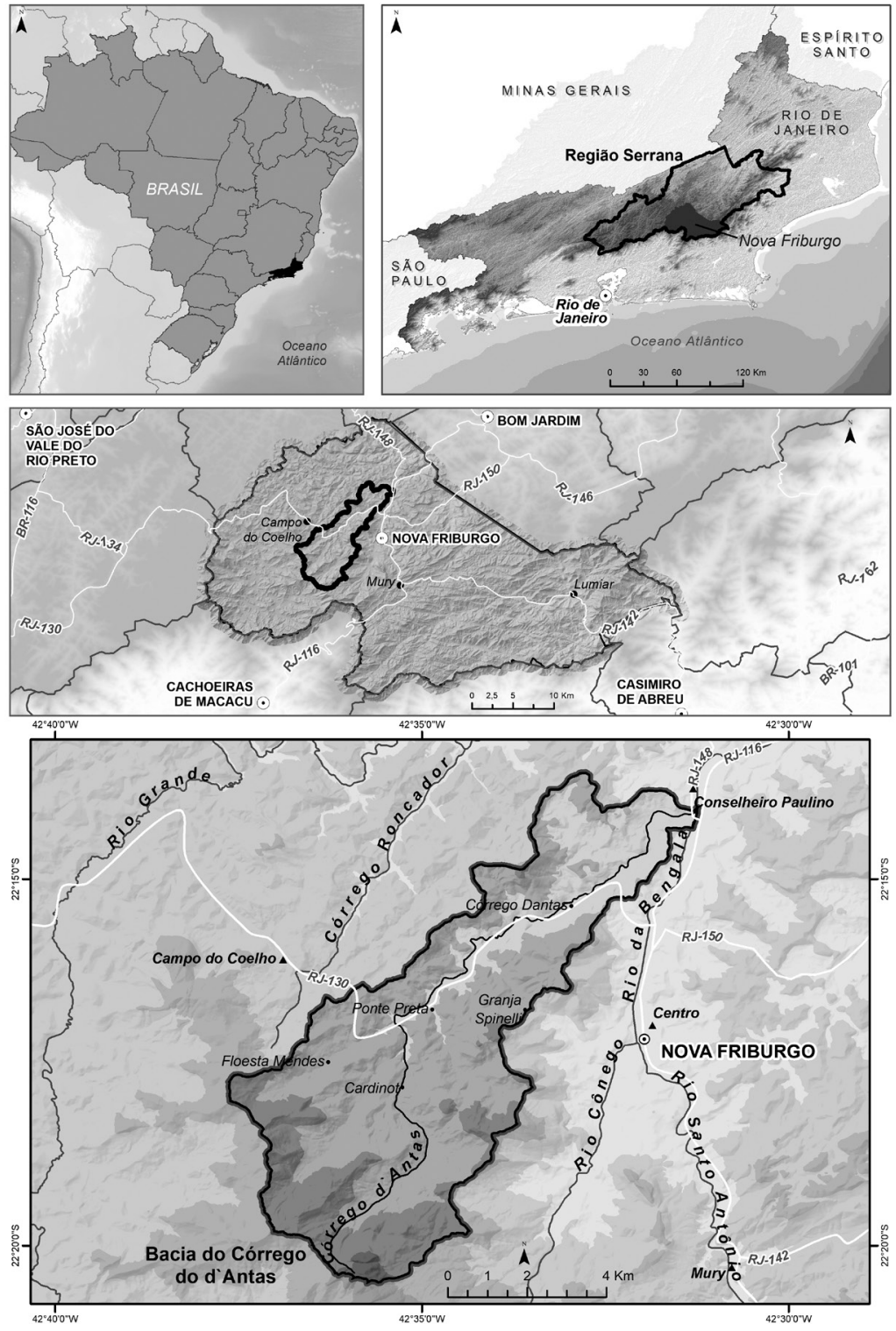

Fonte: Nunes ${ }^{18}$

A Reger-CD parte do pressuposto que a implantação de modelos de GRD requer políticas e ações articuladas de universidades, gestores públicos, sociedade civil organizada e comunidades vulneráveis. Mesmo em contextos de conflitos entre esses setores da sociedade, como ocorreu em diferentes momentos ${ }^{26}$, o enfoque de diálogo entre saberes foi buscado, com vistas à redução das vulnerabilidades sociais e institucionais e ampliação da capacidade de resposta da sociedade e dos órgãos públicos ${ }^{16,26}$.

O presente estudo, desenvolvido a partir da dissertação de mestrado e da pesquisa de pós-doutorado dos autores, tem o objetivo de discutir o processo de construção e utilização do SIG da Reger-CD para apoiar a implantação 
e a consolidação do modelo integrado de gestão local participativa de riscos na bacia do Córrego d'Antas.

\section{Material e métodos}

A base teórico-metodológica para a elaboração deste trabalho é a pesquisa-ação, que pressupõe uma relação entre pesquisador e objeto científico como parte da atuação prática do pesquisador ${ }^{27,28}$. Outra importante base é o conceito de Ecologia de Saberes, que valoriza as formas de conhecimento científico e popular e reconhece a importância da interação entre esses diferentes tipos de conhecimento para produzir novos conhecimentos ${ }^{29}$.

A construção da Reger-CD tem sido feita a partir da realização de seminários com pesquisadores, gestores públicos e moradores de comunidades, apoiados por metodologias de planejamento estratégico-situacional que possibilitam discussão ampla e estruturação de estratégias em busca de soluções para problemas concretos. Foram realizados quatro seminários entre novembro de 2014 e março de 2016, a partir dos quais se definiram ações e produtos específicos a serem construídos; inclusive a construção do SIG de apoio às ações da Reger-CD. Cinco pesquisadores têm trabalhado diretamente na coordenação dessa rede, além de um conjunto de, pelo menos, seis representantes de comunidades. Sem esse trabalho de gestão, a construção do SIG seria muito mais difícil e menos abrangente.

\section{Organização e sistematização do SIG de apoio às atividades da Reger-CD}

A construção do SIG foi orientada para apoiar as atividades da Reger-CD, por meio de análises espaciais e disponibilização de informações sobre o território. Para a manipulação das informações e dados que compõem esse sistema, utilizou-se o software ArcGis 10.4.1, que serviu como plataforma para integrar, editar e disponibilizar as informações espaciais.
Um pesquisador foi responsável por organizar o SIG em seu formato atual. Esse trabalho fez parte da elaboração de sua dissertação de mestrado (durante um ano, dedicou-se cerca de 6 horas semanais para a construção do SIG). Esse pesquisador atuou com base em trabalhos desenvolvidos por outros pesquisadores, em especial, em uma pesquisa de pós-doutoramento e em uma pesquisa de mestrado.

Em função da facilidade de acesso e manipulação, foi utilizado também o software Google Earth como plataforma para disponibilização de informações.

A primeira etapa de construção do SIG constituiu na pesquisa e no levantamento de dados e informações pertinentes à GRDES na bacia hidrográfica do Córrego d'Antas e entorno disponíveis em sítios da internet de instituições oficiais e em instituições parceiras da Reger-CD, como o Laboratório de Geohidroecologia (Geoheco/Universidade Federal do Rio de Janeiro - UFRJ) ${ }^{30}$ e a Prefeitura Municipal de Nova Friburgo.

Após a aquisição dos dados geográficos, os arquivos foram recortados para as áreas de abrangências segundo sua escala de representação cartográfica; e, em alguns casos, suas informações tabulares foram editadas. Os arquivos vetoriais resultantes sofreram procedimentos de transformações cartográficas para adequação em um sistema geodésico de projeção único (UTM 23S - Sistema de Referência Geocêntrico para as Américas -Sirgas2000).

Os dados foram organizados em uma estrutura de diretórios de maneira hierárquica, subdividida segundo critérios de escala e projetos específicos em Conjuntos de Dados Geoespaciais (CDG).

O processo de ordenamento dos arquivos foi realizado com o apoio da extensão ArcCatalog do software ArcGis 10.4.1, utilizada também para renomear cada arquivo vetorial.

O SIG conta com um diretório de documentos referentes aos mapas em formatos PDF e JPG e arquivos dos projetos de mapas próprios do software ArcGis (MXD), que foram produzidos para apoiar as atividades da Reger-CD. 
Esse diretório tem como um de seus objetivos apoiar as estratégias de disponibilização de parte das informações em formatos mais acessíveis a uma maior diversidade de níveis de usuários.

\section{Oficinas de elaboração do 'Plano de Contingência de Base Comunitária e Técnico-Científico da Bacia do Córrego d'Antas' ('Plano de Contingência')}

No SIG da Reger-CD, foram incorporados os dados e as informações espaciais levantados ao longo das 11 oficinas comunitárias, realizadas entre 2016 e 2018 nas localidades de Cardinot, Dois Esquilos, Venda das Pedras e Córrego d'Antas (situadas no interior da bacia do Córrego d'Antas), para a elaboração do 'Plano de Contingência'. Estima-se que 50 pessoas pertencentes a essas comunidades participaram dessas oficinas e contribuíram para a produção das informações.

Nessas oficinas, foram realizados mapeamentos participativos com o objetivo de identificação, pelos moradores, de elementos espaciais que ofereçam entendimentos sobre as fragilidades (pontes que alagam durante chuvas, pessoas com doenças crônicas ou dificuldades de locomoção etc.) e as potencialidades (existência de profissionais da área de saúde, locais onde há tratores e outras máquinas disponíveis etc.) das comunidades relacionadas com os desastres e emergências em saúde, que ajudem a identificar rotas de fugas e abrigos de emergência e formas de monitoramento de eventos relativos à ocorrência de desastres.

Para a realização das atividades de mapeamento, foi utilizado o Google Earth como principal ferramenta. Para tanto, foi necessária a elaboração prévia de um conjunto de dados geográficos que oferecesse aos participantes das oficinas elementos espaciais facilmente identificáveis, fundamentais para auxiliar no mapeamento pretendido.

Para ajudar na familiarização da representação do território da bacia pelo software Google Earth, os participantes das oficinas foram estimulados a localizar suas próprias residências.

A atividade seguinte consistiu na eleição dos elementos espaciais que seriam entendidos como fragilidades e potencialidades. Para tal, foi adotada uma abordagem generalista e flexível, deixando que os próprios participantes apontassem elementos espaciais de acordo com suas interpretações de fragilidades e potencialidades. Após a caracterização desses elementos espaciais, os participantes foram estimulados a localizar e classificar cada um deles, resultando em arquivos KML que, posteriormente, foram organizados e sistematizados para que integrassem o SIG.

O mapeamento seguinte consistiu na localização e na identificação, pelos participantes, de potenciais rotas de fuga e abrigos emergenciais pertinentes à gestão de riscos de chuvas fortes em suas comunidades.

Por fim, foi realizado o levantamento de elementos espaciais e estratégias que apoiassem as atividades de monitoramento das alterações nos níveis dos rios e nos volumes de chuvas nas comunidades.

Posteriormente, as informações levantadas foram sistematizadas e inseridas no SIG. Esse processo de consolidação das informações contou com uma breve análise das informações mapeadas, realizada com base em quatro atividades principais: identificação em campo de parte dos elementos mapeados nas oficinas, conversão dos arquivos em formato KML para Shapefile (SHP) por meio da utilização do software Global Mapper 15.2.3, edição dos arquivos espaciais e ordenamento dos arquivos em diretórios ${ }^{18}$.

\section{Resultados e discussão}

A construção do SIG da Reger-CD é um processo contínuo, na medida em que as novas informações produzidas têm que ser inseridas no sistema, para que ele esteja sempre atualizado e que tenha utilidade real para a GRD. Dessa 
forma, um dos principais resultados obtidos nesta pesquisa-ação é a própria construção coletiva e permanente do SIG.

Outro importante resultado é a utilização do sistema para a realização das ações definidas no âmbito da Reger-CD, com destaque para a execução de pesquisas básicas e aplicadas, a elaboração do 'Plano de Contingência', do 'Atlas de Gestão de Riscos de Desastres do Município de Nova Friburgo e da Bacia Hidrográfica do Córrego d'Antas' ('Atlas') e de diversas ações de formação de alunos do ensino médio que vivem em áreas vulneráveis da bacia do Córrego d'Antas ${ }^{26}$.

Merecem destaque o 'Plano de Contingência', pelo potencial de se tornar uma ferramenta para nortear a atuação dos moradores e do poder público na GRD na bacia do Córrego d'Antas; e o 'Atlas', que terá a abrangência municipal e se tornará o primeiro atlas escolar do Brasil voltado para a discussão de GRD. Quando estiver pronto, será distribuído para todas as escolas públicas de Nova Friburgo, de modo a se tornar um catalisador da discussão sobre
GRD no município a partir das crianças, jovens, responsáveis e professores.

\section{A construção da Base de Dados Geoespaciais vinculada ao SIG da Reger-CD}

Um resultado importante da pesquisa-ação foi a própria construção da Base de Dados Geoespaciais (BDG) (figura 2), que, atualmente, possui 36,7 GB, distribuídos em 6 CDG e um diretório de mapas, que englobam 2.544 arquivos organizados em 71 pastas. Tendo em vista que a maior parte das informações se relaciona com a cartografia básica oficial, a validade delas depende do processo de atualização dos próprios órgãos oficiais. Por outro lado, as informações temáticas relacionadas com a susceptibilidade aos processos hidroerosivos e cobertura vegetal e usos do solo, por exemplo, poderão ser atualizadas à medida que novos mapeamentos sobre esse tema sejam disponibilizados para a Rede. Já as informações levantadas com os moradores só podem ser atualizadas na relação direta com eles ${ }^{16,26}$.

Figura 2. Apresentação esquemática da estrutura da BDG da Reger-CD segundo seus 6 CDG, além do diretório de mapas e figuras

ArcCatalog - D:|BDG_REGER_CD $\quad-\quad \square \quad \times$

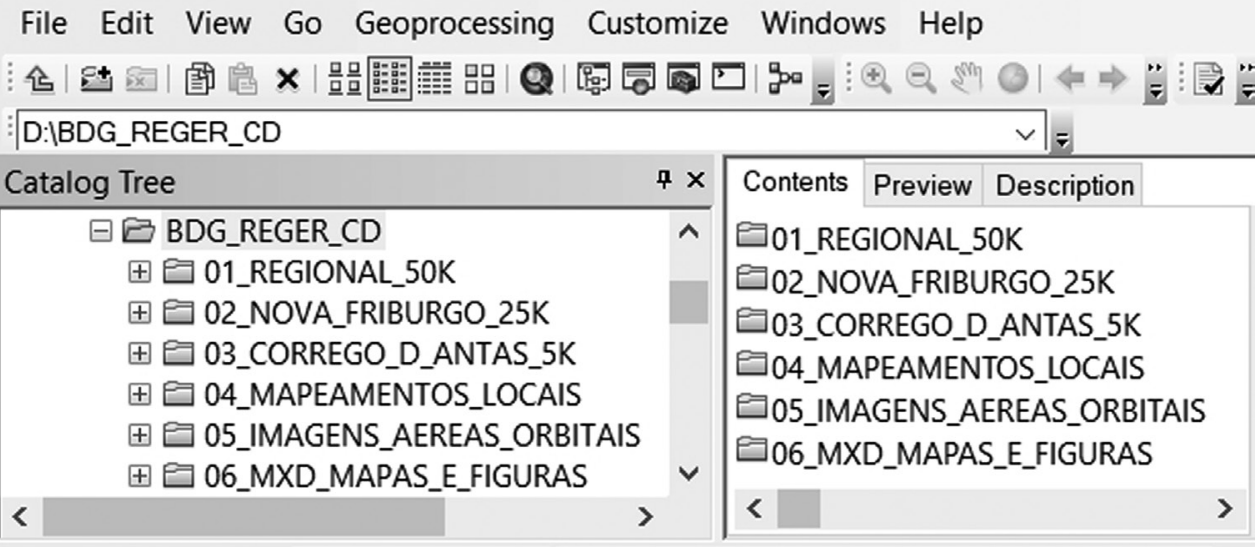

Folder selected 
O SIG inclui informações diversas que ganham potência quando são relacionadas, pois permitem realizar análises específicas importantes para a gestão. Por exemplo, saber onde estão as Unidades de Saúde mais próximas da bacia do Córrego d'Antas é importante, todavia, relacionar essa informação com as pessoas que demandam cuidados de saúde específicos possibilita desenhar respostas voltadas para esses grupos. Conhecer a localização de abrigos de emergência é essencial, mas relacionar essa informação com as áreas mais suscetíveis a deslizamentos e a inundações permite pensar políticas públicas de alerta e alarme mais adequadas.
Essas relações entre camadas de informações são passíveis de serem realizadas para os três recortes espaciais integrados e para as três escalas cartográficas para as quais há informações inseridas na BDG (figura 3): o CDG da escala de 1:50.000 agrega informações relativas ao recorte espacial dos municípios de Teresópolis, Petrópolis e Nova Friburgo; o CDG da escala 1:25.000 é composto por informações sobre o território de Nova Friburgo; e finalmente, o CDG da escala de 1:5.000 reúne informações do interior da bacia hidrográfica do Córrego d'Antas.

Figura 3. Apresentação esquemática das áreas de abrangência dos Conjuntos de Dados Geográficos, com suas escalas de referências, da BDG da Reger-CD

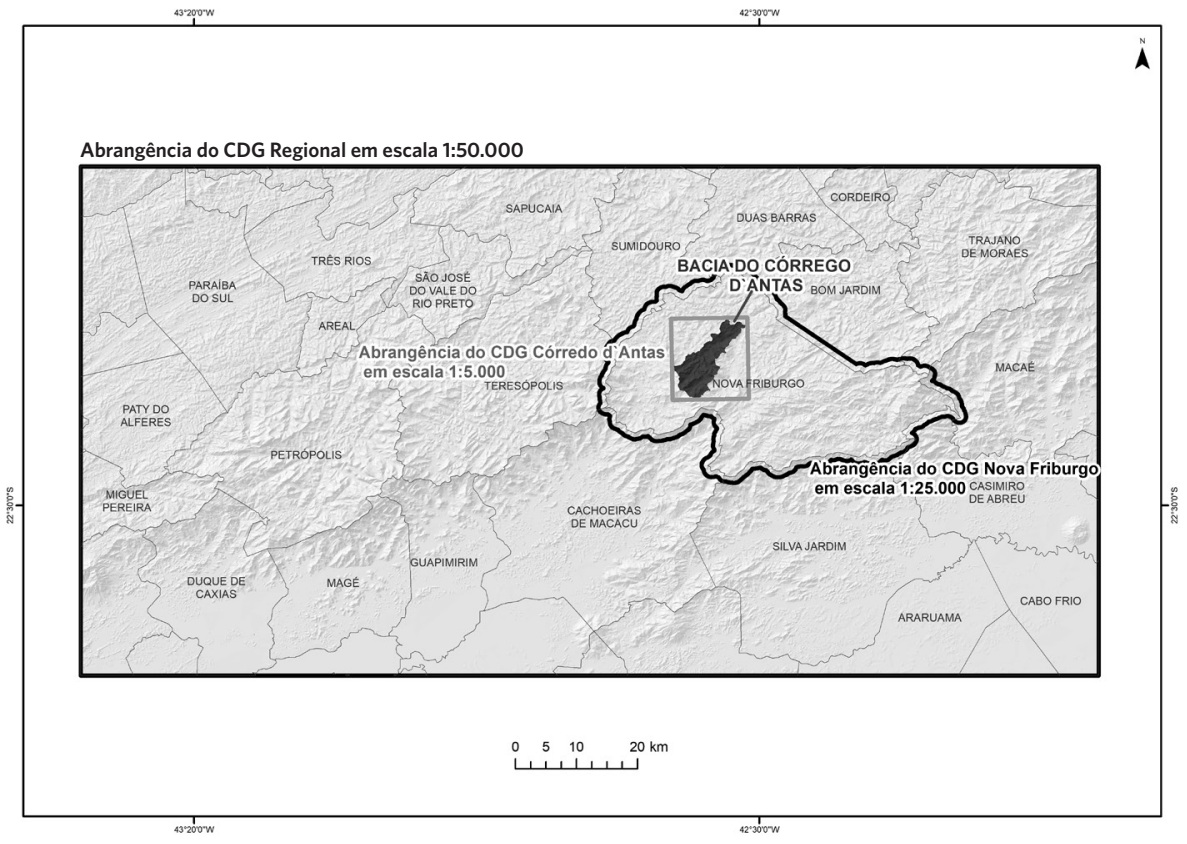

Fonte: Elaboração própria.

Cada uma dessas escalas e recortes espaciais é importante para agregar determinadas informações e atores que impactam sobre a produção de distintas análises de vulnerabilidade aos desastres e emergências em saúde e de capacidades de resposta da sociedade e do poder público.

Nesse sentido, as escalas mais locais, como o CDG da escala 1:5.000, oferecem visibilidade a atores e dinâmicas sociais que 
dificilmente são observadas nas escalas de mapeamento da maior parte das políticas públicas estaduais e municipais.

Da mesma forma, o mapeamento em diferentes escalas articuladas permite a formulação de propostas de atuação integradas, porém diferentes.

\section{CDG 1:50.000}

Esse CDG incorpora informações para representação cartográfica de dados em escalas originais de obtenção da informação geográfica próximas a 1:50.000 (intervalo da escala de representação cartográfica entre 1:25.000 e 1:100.000, com generalizações). Os dados que o integram compõem uma visão regional para a GRDES. Portanto, trata-se de uma escala cartográfica de análise capaz de apoiar ações de vigilância em saúde em nível regional e municipal.

Nesse conjunto de dados, é possível acessar, por exemplo, o mapa de susceptibilidade a deslizamentos rasos para Teresópolis, Petrópolis e Nova Friburgo, assim como a localização de todas as US existentes nesse último município.

Nessa escala de análise, portanto, foi possível relacionar a localização das US de Nova Friburgo com o mapa de suscetibilidade aos movimentos de massa e entender quais unidades estão em locais de elevada suscetibilidade a esses eventos, fator-chave para análise da vulnerabilidade institucional do setor saúde aos desastres. Inclusive, esse relacionamento de informações foi base para uma pesquisa de mestrado realizada no âmbito da Reger- $\mathrm{CD}^{31}$, cujos resultados são importantes para implantação de políticas públicas do setor saúde.

\section{CDG 1:25.000}

Este CDG reúne arquivos disponibilizados pelo Instituto Brasileiro de Geografia e Estatística (IBGE) referentes ao mapeamento sistemático para a escala 1:25.000 do estado do Rio de Janeiro e arquivos selecionados do conjunto de dados disponibilizado pelo Geoheco/ $\mathrm{UFRJ}^{30}$. Agrega informações regionais para representação cartográfica de dados em escalas originais de obtenção da informação geográfica próximas a 1:25.000 (intervalo da escala de representação cartográfica entre 1:10.000 e 1:50.000, com generalizações).

Suas informações e dados objetivam apoiar a GRDES por meio da oferta de uma cartografia básica oficial, atual e de ampla utilização.

Esse conjunto permite análises espaciais referentes aos aspectos geobiofísicos, de infraestrutura, sistema viário e político administrativo da bacia hidrográfica do Córrego d’Antas e de Nova Friburgo.

Esse CDG oferece o que há de mais atual em dados espaciais produzidos sobre a rede hidrográfica, o relevo e as infraestruturas da bacia e do município, apoiando a gestão de riscos com informações sobre as redes saúde, segurança pública, educacional e viária etc.

Também é possível acessar, nesse CDG, mapeamentos de suscetibilidade a deslizamentos rasos e de cobertura vegetal e uso da terra, tanto para o território da bacia do Córrego d'Antas quanto para o município de nova Friburgo, oferecendo uma perspectiva que integra a gestão de riscos na bacia ao recorte territorial municipal. É adequada, portanto, para apoiar ações de vigilância em saúde no recorte espacial municipal, dos distritos de Nova Friburgo, bairros e bacias hidrográficas.

Tendo em vista a atualidade dos dados, o seu caráter oficial, a escala de detalhes, o recorte espacial das informações e a qualidade dos dados temáticos elaborados, esse CDG representa o conjunto de dados geográficos mais utilizado como apoio à gestão de riscos da Reger-CD, tendo sido empregado para a elaboração de materiais de apoio às oficinas do 'Plano de Contingência', ao 'Atlas' e à elaboração de pesquisa sobre a adequabilidade dos abrigos emergenciais situados na bacia do Córrego d'Antas ${ }^{32}$. Na perspectiva da ecologia de saberes, esse CDG, com o anterior, pode ser entendido como parte dos saberes institucionais sobre o território, oferecendo à GRDES o conhecimento oficial disponibilizado pelos órgãos públicos e pelo saber científico. 


\section{CDG 1:5.000}

O CDG dessa escala agrupa informações locais para representação cartográfica de dados em escalas originais de obtenção da informação geográfica próximas a 1:5.000 (intervalo da escala de representação cartográfica sugerido entre 1:2.000 e 1:10.000, podendo haver generalizações).

Tendo em vista que esse CDG é basicamente produto da organização de dados e informações produzidas por pesquisas cientificas e que sua cartografia básica não está incluída nos mapeamentos sistemáticos oficiais, esse é entendido como o conjunto que mais representa o apoio institucional científico ao SIG da Reger-CD.

As informações disponibilizadas nesse conjunto são próprias para a gestão local na bacia do Córrego d'Antas - adequado, principalmente, para o planejamento territorial urbano. As cartografias básica e temática desse CDG oferecem informações sobre o modelado do terreno, rede de canais e rede viária, além de mapeamentos de suscetibilidade de deslizamentos rasos e de cobertura vegetal e usos da terra de maior riqueza de detalhes; fundamentais para a GRDES nas escalas locais. A escala mais próxima também permite um planejamento do território e o apoio às ações de vigilância em saúde em recortes mais específicos, como dos bairros e localidades inseridas na bacia.

Esse conjunto de dados, portanto, encerra o maior potencial para servir de pano de fundo aos mapeamentos locais. Está sendo utilizado para subsidiar a elaboração dos encartes de maior detalhe do 'Plano de Contingência', permitindo a produção de mapas de gestão detalhados, como os de abrigos emergenciais e rotas de fuga. Também apoia a elaboração do 'Atlas' (a sua unidade referente à bacia do Córrego d'Antas). Foi essencial ainda para a elaboração do estudo sobre abrigos emergenciais referido acima ${ }^{34}$, que utilizou de forma integrada elementos do CDG 1:25.000 e 1.5.000.

\section{CDG Mapeamentos Locais}

Este CDG agrupa informações locais para representação cartográfica de dados em escalas originais de obtenção da informação geográfica próximas a 1:500 ou mais detalhadas. As informações nele inseridas foram produzidos a partir de atividades de campo ou nas oficinas participativas do 'Plano de Contingência'.

Dessa forma, esse CDG integra ao SIG da Reger-CD os saberes comunitários sobre o território, representando o resultado de investigações sobre as percepções dos moradores da bacia adiante de gestão de riscos.

Tendo em vista o caráter das informações sistematizadas, esse CDG é entendido como o conjunto mais flexível ante os rigores cartográficos formais e, portanto, o mais aberto à inserção de informações espontâneas e de temáticas que fogem às categorizações utilizadas nos CDG anteriormente apresentados.

A alimentação contínua de informações nesse conjunto pode oferecer à GRDES, no âmbito da Rede, uma ferramenta dinâmica e de empoderamento do conhecimento local, fundamentais para enriquecer uma gestão participativa e com maior cognição local.

As informações contidas nesse CDG têm foco específico na GRD relacionados com as chuvas fortes e/ou contínuas. Relacionamse com as percepções dos moradores locais sobre as fragilidades, potencialidades, rotas de fuga, abrigos de emergência e estratégias de monitoramentos de rios e do volume de chuvas em suas comunidades. As figuras $4 e$ 5 ilustram mapeamentos participativos realizados, apresentando informações contidas no CDG Mapeamentos Locais já consolidadas e disponibilizadas em formato para utilização no software Google Earth. A primeira mostra uma camada de informações relativa às áreas e pessoas vulneráveis, enquanto a última apresenta informações relacionadas com o monitoramento de chuva e rios. 
Figura 4. Mapa de distribuição das áreas e pessoas vulneráveis mapeadas durante as oficinas de mapeamento participativo

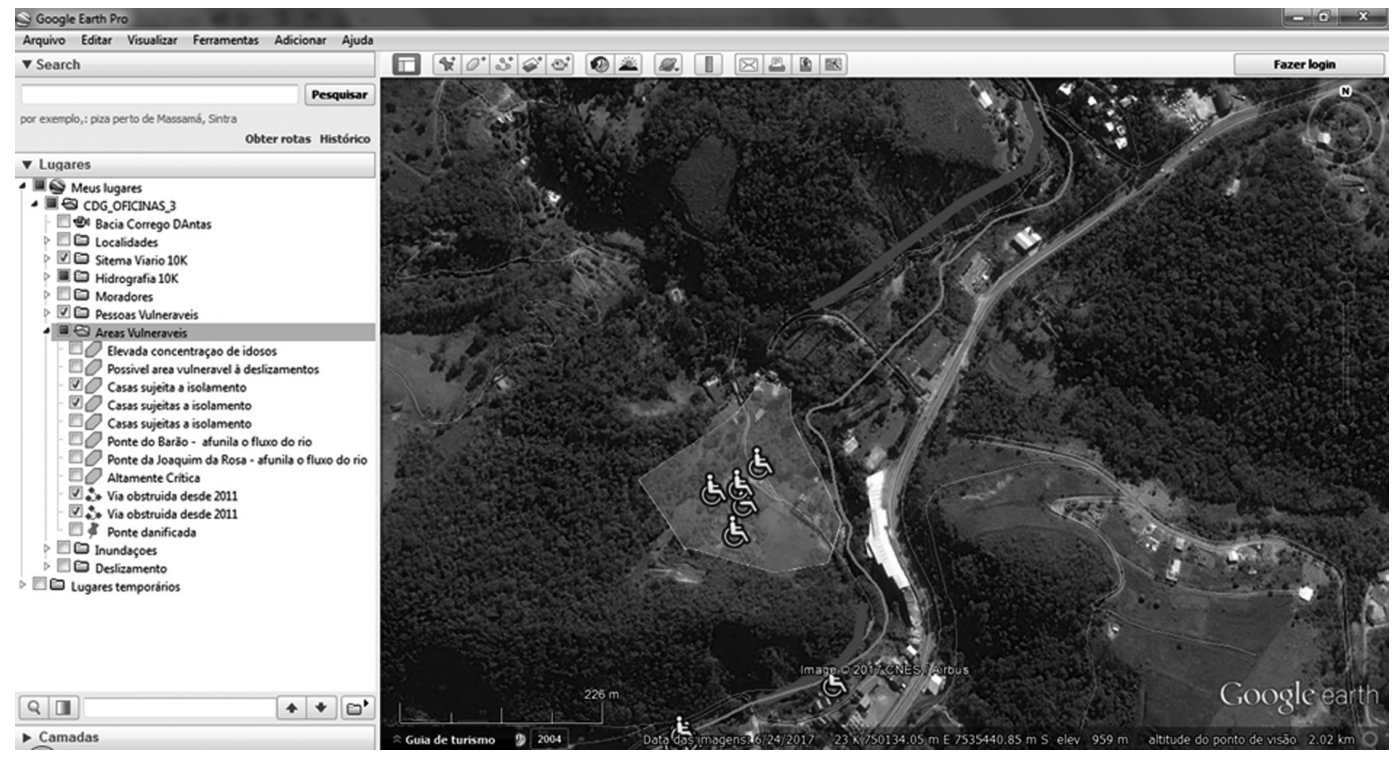

Fonte: Elaboração própria.

Figura 5. Mapa de distribuição das áreas utilizadas para observação do volume de rios e chegada de chuvas mapeadas durante as oficinas de mapeamento participativo

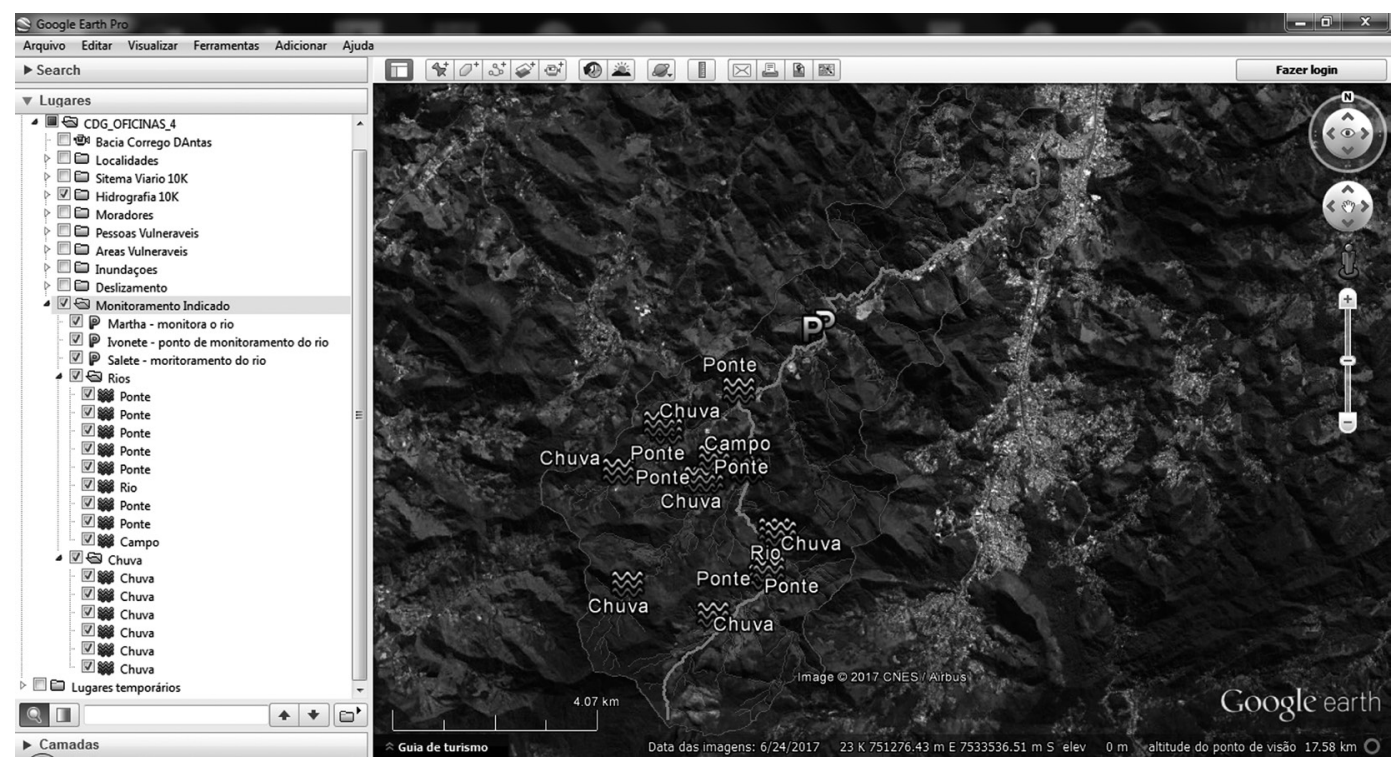

Fonte: Elaboração própria.

Essas informações têm sido essenciais para a elaboração do 'Plano de Contingência' da bacia e para a definição de estratégias integradas de RRD que incluem desde a geração do conhecimento até os processos de recuperação e reabilitação. 


\section{Considerações finais}

A construção de uma rede institucional e comunitária é uma alternativa que possui potencial para apoiar processos consistentes de $\mathrm{RRD}$, especialmente por fortalecer uma cultura de GRDES, mantendo a temática em contínua discussão, e por fortalecer o diálogo entre os diversos atores envolvidos com essa temática.

Na prática, porém, esse processo é difícil e conflituoso, pois os saberes técnico-científicos possuem uma legitimidade social para discutir a GRDES que os saberes populares não têm. Portanto, a construção de uma rede institucional e comunitária que busque um diálogo horizontal entre saberes requer, essencialmente, uma atitude de humildade dos pesquisadores e gestores públicos perante os moradores locais. Atitude esta que não é parte da cultura das instituições acadêmicas e de gestão.

Além disso, requer um empoderamento dos moradores locais para que expressem seus conhecimentos e, a partir destes, participem ativamente das discussões e atividades relacionadas com a GRDES.

A construção participativa de um SIG é uma ferramenta com grande potencial para apoiar esse diálogo entre saberes, pois necessita dos saberes técnico-científicos e locais para que seja capaz de qualificar a gestão de riscos.

A construção do SIG da Reger-CD mostrou-se capaz de apoiar decisivamente esse diálogo, mesmo em um contexto no qual havia conflitos importantes entre poder público e moradores locais ${ }^{\mathbf{2 6}}$.

Além desse papel, esse SIG tem-se mostrado uma ferramenta para apoiar o conjunto de processos relacionados com a GRD, conforme discutidos por Narváez et al.7 e recomendados pelo Marco de Sendai.

Mesmo que o processo de mapeamento participativo tenha ocorrido no âmbito da Rede, o levantamento de informações encontrou dificuldades importantes; uma delas concernente à mobilização dos moradores para a participação. A despeito de ter havido um número significativo de moradores nas oficinas, elas contaram com poucos moradores provenientes de algumas áreas de maior densidade populacional e de áreas rurais mais isoladas. Isso gerou uma limitação da base para a GRD. Essa limitação dificulta que se avance como desejado em direção ao cumprimento do ODS 11, pois impossibilita o levantamento de informações locais para o conjunto da bacia a partir de um diálogo entre saberes acadêmicos e populares. Dificulta também o avanço em direção à prioridade estabelecida pelo Marco de Sendai de promover acesso em tempo real a dados confiáveis (isso ocorreu para algumas áreas, mas não para outras) e também de utilizar inovações em tecnologia da informação e comunicação para melhorar as ferramentas de medição, coleta, análise e divulgação de dados.

Para o processo de geração de conhecimento, o SIG é fundamental, pois, ao disponibilizar as informações existentes, possibilita entender quais informações ainda precisam ser produzidas.

Para a redução de riscos atuais e futuros, as informações contidas no SIG são essenciais, na medida em que possibilitam, por exemplo, que todos conheçam as áreas suscetíveis aos desastres e as populações vulneráveis, apoiando processos de redução das vulnerabilidades.

Na preparação de respostas aos desastres e emergências em saúde, são muitas as utilidades do SIG. Conhecer as pessoas vulneráveis e as capacidades de resposta das comunidades e do poder público e poder relacionar ambas, por exemplo, é essencial. Permite planejar as ações emergenciais priorizando populações vulneráveis e otimizando a capacidade de resposta, fundamental para reduzir mortes e agravos ${ }^{8}$.

No que tange à resposta e reabilitação e à recuperação e reconstrução, conhecer as áreas mais suscetíveis a alagamentos e movimentos de massa possibilita um planejamento territorial mais adequado e que reduza os riscos futuros.

O SIG da Reger-CD, portanto, tem potencial para atuar de diversas formas, apoiando o conjunto de processos relacionados com a GRDES na bacia do Córrego d'Antas. 
Não obstante, dificuldades têm sido encontradas para o desenvolvimento desse sistema, especialmente no que concerne à sua disponibilização como uma ferramenta de uso amplo; particularmente por questões técnicas, relacionadas com o armazenamento da BDG construída no âmbito desse sistema. Afinal, a Reger-CD não possui recursos financeiros suficientes para construir um sistema robusto, disponível on-line, que possa ser facilmente acessado. Vencer esse desafio é fundamental para tornar essa base uma ferramenta de uso público capaz de apoiar todos os processos de GRDES na bacia.

\section{Referências}

1. Organização das Nações Unidas. Agenda 2030 [internet]. [acesso em 2019 jul 12]. Disponível em: http:// www.agenda2030.org.br/ods/11/.

2. Gould SJ. Time`s arrow, time`s cycle: Myth and Metaphor in the Discovery of Geological Time. Massachusetts: Harvard University Press; 1987.

3. Ludwig L, Mattedi MA. As tecnologias da informação e comunicação na gestão dos riscos de desastres socioambientais. Ambient. soc. [internet]. 2018 [acesso em 2019 ago 18]; (21):e01034. Disponível em: http:// dx.doi.org/10.1590/1809-4422asoc0103r4vul8liao.

4. Mattedi MA, Butzke IC. A Relação entre o social e o natural nas abordagens de hazards e de desastres. Ambient. soc. 2001; 9(2):10-21.
Outro desafio está no processo de atualização constante do SIG, necessário para que ele não fique desatualizado e torne-se pouco útil. Construir mecanismos colaborativos de atualização dos dados e informações contidos na BDG é essencial.

\section{Colaboradores}

Freitas LE (0000-0003-4751-356X)* e Nunes FSB (0000-0002-5784-5819)* contribuíram igualmente para a elaboração do manuscrito.
5. Brasil. Ministério da Integração Nacional, Secretaria Nacional de Proteção e Defesa Civil. Módulo de formação: noções básicas em proteção e defesa civil e em gestão de riscos: livro base. Brasília, DF: Ministério da Integração Nacional; 2017.

6. Freitas LE, Fernandes R, Coelho Netto AL. Post-catastrophic disaster induced laws for climatic change adaptation: a case study in SE-Brazil. In: Leal Filho W, Nagy G, Borga M, et al., organizadores. Climate Change, Natural Hazards and Adaptation Option: Handling the impacts of a changing climate. Hamburgo: Springer International Publishing; 2020. p. 197-212.

7. Narváez L, Lavell A, Pérez G. La gestión del riesgo de desastres: un enfoque basado en procesos. San Isidro: Secretaría General de la Comunidad Andina; 2009.
*Orcid (Open Researcher and Contributor ID). 
8. United Nations Office for Disaster Risk Reduction. Sendai Framework for Disaster Risk Reduction 2015 - 2030 [internet]. [local desconhecido]: United Nations; 2016. [acesso em 2019 jul 18]. Disponível em: https://www.unisdr.org/files/43291_sendaiframeworkfordrren.pdf.

9. Freitas CM, Mazoto ML, Rocha V. Guia de preparação e resposta do setor saúde aos desastres. Rio de Janeiro: Fiocruz; Secretaria de Vigilância em Saúde; 2018.

10. Agencia Suiza para el Desarrollo y la Cooperación. Instrumentos de apoyo para el análisis y la gestión de riesgos naturales: Guía para el especialista [internet]. Managua: 2002. [acesso em 2019 ago 16]. Disponível em: http://www.snet.gob.sv/Riesgo/GuiaMetodologica.pdf.

11. Peru. Ministerio de Economía y Finanzas del Perú; Estrategia Internacional para la Reducción de Desastres de la ONU. Memoria del taller internacional Lecciones Aprendidas de la Gestión del Riesgo en Procesos de Planificación e Inversión para el Desarrollo. Lima: Agencia Suiza para el Desarrollo y la Cooperación; 2011.

12. Câmara G, Medeiros CB, Casanova MA, et al. Anatomia de Sistemas de Informação Geográfica [internet]. São Paulo: Inpe; 1996. [acesso em 2017 dez 2]. Disponível em: http://www.dpi.inpe.br/geopro/livros/anatomia.pdf.

13. Marcelino EV. Desastres naturais e geotecnologias: conceitos básicos. Santa Maria: INPE; 2008. [Série Caderno Didático n. 1].

14. Abdula A, Taela K. Avaliação das capacidades de gestão do risco de desastres em Moçambique: Netherlands Climate Change Studies Assistance Programme Phase I. Maputo: Ministério para a Coordenação da Acção Ambiental; 2005.

15. Avila MRR, Mattedi MA. Desastre e território: a produção da vulnerabilidade a desastres na cidade de Blumenau/SC. Rev. Bras. Gestão Urbana. Curitiba. 2017; 9(2):187-202.
16. Freitas LE, Coelho-Netto AL. Reger Córrego d'Antas: uma ação coletiva para enfrentamento de ameaças naturais e redução de desastres socioambientais. Ciênc. Trópico. 2016; (40):165-190.

17. Ferreira D. Sistema de Informações Geográficas Participativo (SIG-P) na Prevenção de Desastres Ambientais: estudo de caso do Morro do Baú em Ilhota/SC [dissertação]. Florianópolis: Universidade do Estado de Santa Catarina; 2012. 168 p.

18. Nunes FSB. Base de dados geoespaciais no apoio à gestão participativa de riscos de desastres: o caso da bacia hidrográfica do Córrego d'Antas - Nova Friburgo/RJ. [dissertação]. Rio de Janeiro: Universidade Federal Rural do Rio de Janeiro; 2018. 162 p.

19. Weiner D, Harris TM, Craig WJ. Community Participation and Geographic Information Systems [internet]. 2001. [acesso em 2016 jan 6]. Disponível em: http://www.iapad.org/wp-content/uploads/2015/07/ Community-Participation-and-Geographic-Information-Systems.pdf.

20. Freitas LE, Sato AM, Schottz S, et al. Community, University and Government Interactions for Disaster Reduction in the Mountainous Region of Rio de Janeiro, Southeast of Brazil. In: Leal Filho W, Azeiteiro UM, Alves F, organizadores. Climate Change and Health: Improving Resilience and Reducing Risks. Nova York: Springer International Publishing; 2016. p. 313-328.

21. Freitas LE, Cruz JCHO, Cortines AC, et al. Observatory of Sustainable and Healthy Territories (OTSS) GIS: Geo-Information for the Sustainability of Traditional Communities in Southeastern Brazil. In: Leal Filho W, Azeiteiro UM, Alves F, organizadores. Climate Change and Health Improving Resilience and Reducing Risks. led.Nova York: Springer International Publishing; 2016. p. 353-367.

22. Cinderby S. Participatory Geographic Information Systems (GIS): the future of environmental GIS? Int J Environ Pollution [internet]. 1999 [acesso em 2014 dez 16]; 11(3):304-315. Disponível em: https:// www.researchgate.net/publication/249919805_Ge- 
ographic_Information_Systems_GIS_for_Participation_The_Future_of_Environmental_GIS.

23. Viana VJ. Gestão de riscos de desastres no Brasil: Leitura das estratégias locais de redução de risco de inundação em Nova Friburgo, RJ [tese]. Rio de Janeiro: Universidade do Estado do Rio de Janeiro; 2016. $407 \mathrm{p}$.

24. Coelho Netto AL, Sato AM, Avelar AS, et al. January 2011: The Extreme Landslide Disaster in Brazil. In: Margottini C, Canuti P, Sassa K, organizadores. Landslide Science and Practice. Berlin: Springer Berlin Heidelberg; 2013. p. 377-384.

25. Fundação Coppetec, Laboratório de Hidrologia e estudos de Meio Ambiente. Elaboração do Plano Estadual de Recursos Hídricos do Estado do Rio de Janeiro R3-A - Temas técnicos estratégicos RT-03 - Vulnerabilidade a Eventos Críticos. Ocorrências de Desastres Naturais entre 2000 e 2012 por Região Hidrográfica [internet]. Rio de Janeiro: Coppetec; 2014. [acesso em 2016 jul 2]. Disponível em http://www.inea. rj.gov.br/cs/groups/public/documents/document/ zwew/mdyy/ edisp/inea0062130.pdf.

26. Freitas LE, Coelho Netto AL. Gestão de riscos de desastres e participação popular: lições aprendidas e a relevância da educação para a consolidação da Rede de Gestão de Riscos da Bacia Hidrográfica do Córrego d'Antas (Reger-CD), Nova Friburgo/RJ. Rev Geo Colégio Pedro II. 2019; (4):89-101.

27. Engel GI. Pesquisa-ação. Edu. Revista. 2000; (16):181-191.
28. Thiollent M. Metodologia da pesquisa-ação. São Paulo: Cortez; 2006.

29. Santos BS. Para além do pensamento abissal: das linhas globais a uma ecologia de saberes. Novos estud. - CEBRAP. 2007; (79):71-94.

30. Coutinho BH. Indicadores Geo-Hidroecológicos de Susceptibilidade das Encostas frente à erosão e movimentos de massa em Região Montanhosa Tropical Úmida: suporte metodológico para zoneamentos de susceptibilidade e risco em diferentes escalas de análise espacial. Relatório Final de Pós-Doutorado. Rio de Janeiro: UFRJ; 2014.

31. Vida IM. Vulnerabilidade institucional do setor saúde a desastres no município de Nova Friburgo [dissertação]. Rio de Janeiro: Fundação Oswaldo Cruz; 2019.

32. Duek TCN, Freitas LE, Mendonça MB. Preliminary evaluation of emergency shelters for disasters associated with landslides at the hydrographic basin of Corrego d'Antas, Nova Friburgo, Rio de Janeiro, Brazil. In: Leal Filho W, Nagy G, Borga M, et al., organizadores. Climate Change, Natural Hazards and Adaptation Option: Handling the impacts of a changing climate. Hamburgo: Springer International $\mathrm{Pu}-$ blishing; 2020. p. 177-195.

Recebido em 29/09/2019

Aprovado em 29/04/2020

Conflito de interesses: inexistente

Suporte financeiro: não houve 